\title{
Transforaminal percutaneous endoscopic lumbar discectomy for upper lumbar disc herniation: clinical outcome, prognostic factors, and technical consideration
}

\author{
Yong Ahn • Sang-Ho Lee • June Ho Lee • Jin Uk Kim • \\ Wei Chiang Liu
}

Published online: 21 July 2009

(C) Springer-Verlag 2009

\section{Erratum to: Acta Neurochir}

DOI 10.1007/s00701-009-0204-x

The correct Table 3 is given here.
The online version of the original article can be found at http://dx.doi. org/10.1007/s00701-009-0204-x.

\section{Y. Ahn}

Department of Neurosurgery, Wooridul Spine Hospital,

Daegu 700-732, South Korea

\section{S.-H. Lee • J. H. Lee}

Department of Neurosurgery, Wooridul Spine Hospital,

Seoul, South Korea

\section{J. U. Kim}

Department of Orthopedic Surgery, Wooridul Spine Hospital,

Seoul, South Korea

W. C. Liu

Department of Radiology, Wooridul Spine Hospital,

Seoul, South Korea

\section{Y. Ahn $(\bowtie)$}

Wooridul Spine Hospital,

50-3 Dongin-dong, Jung-gu,

Daegu 700-732, South Korea

e-mail: ns-ay@hanmail.net
Table 3 Clinical factors affecting surgical outcome ( $N=45$ patients)

\begin{tabular}{llll}
\hline Variables & $\begin{array}{l}\text { Excellent } \\
\text { group }\end{array}$ & $\begin{array}{l}\text { Non-excellent } \\
\text { group }\end{array}$ & $p$-value \\
\hline Age $\geq 45$ years & 12 & 21 & $0.041^{\mathrm{b}}$ \\
Age $<45$ years & 9 & 3 & \\
Male & 18 & 15 & 0.077 \\
Female & 3 & 9 & \\
Symptom duration $<6$ mo & 13 & 7 & $0.038^{\mathrm{b}}$ \\
Symptom duration $\geq 6$ mo & 8 & 17 & \\
Back pain dominant & 4 & 13 & 0.252 \\
Leg pain dominant & 4 & 4 & \\
Leg pain and back pain & 13 & 19 & 0.143 \\
Motor weakness $(+)$ & 2 & 7 & \\
Motor weakness $(-)$ & 19 & 17 & 1.000 \\
Reverse Lasegue's sign $(+)$ & 6 & 7 & 0.782 \\
Reverse Lasegue's sign $(-)$ & 15 & 17 & \\
Operation time ${ }^{\text {a }}$ (min) & $62.9 \pm 27.8$ & $60.4 \pm 19.0$ &
\end{tabular}

Fisher's exact test

${ }^{\text {a }}$ Mann-Whitney $U$ test (mean $\pm \mathrm{SD}$ )

${ }^{\mathrm{b}}$ Statistically significant value 